\title{
Scintillator Crystals: Structure, Characterization and Models for Better Performances
}

\author{
Daniele Rinaldi * (i) and Luigi Montalto \\ Dipartimento di Scienze e Ingegneria della Materia, dell'Ambiente ed Urbanistica (SIMAU), Università \\ Politecnica delle Marche, 60131 Ancona, Italy; 1.montalto@univpm.it \\ * Correspondence: d.rinaldi@staff.univpm.it
}

Received: 4 February 2020; Accepted: 5 February 2020; Published: 7 February 2020

Keywords: scintillating crystals; crystal structure; crystal defects; scintillating properties; scintillating performances; models; quality control; elasto- and electro-optics; crystals characterization

The majority of instrumentation and systems for radiation detection are based on scintillators. Scintillating materials convert high-energy radiation in visible light (visible photons), this light is collected by a photomultiplier and elaborated to obtain information about incident radiation energy spectra and for image reconstruction. Among the variety of scintillating substances and forms, the scintillating crystals offer the best performances in various fields of application. Medicine, Physics, Industry, Earth and Soil science, Aerospace, Astronomy applications and others, require more and more sophisticated and accurate tools to face new and deeper challenges. In this direction, the development of new materials and the improvement of the existing ones is mandatory. A deeper knowledge of the crystal physics as well as all the mechanisms relative to their scintillating behaviour is crucial to accomplish the needs of these applications. Theories and simulations are fundamental to obtain predictive models. Further to these, accurate testing procedures and quality control methodologies play a major role in monitoring the crystal condition and improving the production processes, helping the producers of crystals to enhance the process efficiency and allowing crystals' "end users" to have better performing devices.

Medical instrumentation, for diagnostics and imaging, requires high sensitivity, in addition to a fast and reliable response, thereby reducing health risk and increasing both the spatial and time resolution. The different bio-medical instrumentation spans from the PET, to the high-resolution SPECT and Gamma camera, including X-Ray imaging [1]; therefore, each crystal species must be optimized for the relevant purpose.

However, security and geological instrumentation require another kind of scintillating crystal, which is durable and stable over time.

The design and construction of calorimeters for high-energy physics studies are critical in scintillator development. It is opportune to emphasize the discovery of the Higg's boson at CERN by CMS calorimeter, where about 80,000 large-dimension $\mathrm{PbWO}_{4}(\mathrm{PWO})$ were used. For the first time, a large-scale production of big crystals $(2 \mathrm{~cm} \times 2 \mathrm{~cm} \times 20 \mathrm{~cm})$ was necessary [2,3]. Moreover, the large production scale called for a fast and non-destructive quality control to avoid, among the other quality requirements, the risk of fracture due to internal stress [4]. The implementation of in-line quality control using a fast and non-destructive technique is mandatory for all of the above applications. The different applications have specific requirements, since scintillators must be able to detect photons at different energies. For example, energy detected by PWO crystals at CMS is of the order of $100 \mathrm{Gev}$ and the photon energy for X-Ray imaging decreases down to $20 \mathrm{KeV}$.

Different scintillator parameters are crucial for determining the efficiency and accuracy of detection systems. Among them, we cite some significant parameters such as the following: the Light Yield 
(LY), which evaluates the production light efficiency; LY is expressed as the number of visible photons emitted per unit of deposited energy (conventionally, number of photons/MeV) and varies depending on the crystal variety, surface and crystal condition [5];

The Decay Time is another remarkable parameter, in fact, a fast response is essential in medical imaging and diagnostics as it allows for a lower dose to be administered to the patient, thus decreasing the health risk [6,7]; moreover, the Detection Efficiency is determined by the number of recorded events over the radiation emitted by a source [8], this last parameter is governed by the stopping power, which is the capacity of the scintillator to block the radiation.

The enhancement of these crystal properties implies a theoretical and simulation effort with, in parallel, the development of structural assessment and quality control procedures. In this direction, this Special Issue, entitled "Scintillator Crystals: Structure, Characterization and Models for Better Performances", provides a wide spectrum of contributions from the review of quality control to the simulation and theoretical evaluation of the decay time, reviewing the state-of-the-art of organic and inorganic crystals for different purposes.

The contribution by Montalto et al. [9] provides an updated review of the state-of-the-art regarding crystal quality control. Since performances depend on the crystal quality, the techniques used to analyse the crystal state are explained. First, methods for structural analysis are exposed, based on X-Ray diffraction (XRD) and Electron Microscopy. XRD measurements can be accompanied by morphological and compositional studies by means of High-Resolution Scanning Electron Microscopy (HRSEM). The Transmission Electron Microscopy (TEM), despite being a destructive approach, allows for rapid analysis. In this paper, cross-section TEM analysis of LUAG fibers are shown. Moreover, since the surface influences the Light Yield, a method to assess the surface state is shown, based on Grazing Incidence X-Ray Diffraction (GID). The paper explains the basic photoelastic theory and the Bertin surfaces for the detection of internal stress. Since internal stress is the signature of crystal defects, the paper describes the Conoscopy and Sphenoscopy (both based on photoelasticity) as reliable methods for detecting the stress state of the crystals. They are fast and non-destructive methods that can be used for the purposes of quality control, assessment of the elasto-optic parameters [10], production process enhancement and costs reduction. In particular, the new Sphenoscopic technique is a fast and very easy to apply analysis for quality control.

The paper by Cieślak et al. [11] is a review about the crystals for the detection of radioactive materials. The necessity to detect and discriminate the different sources is fundamental for security, nuclear decommissioning, decontamination, border security, nuclear proliferation and nuclear medicine. For the neutron detection, organic and inorganic scintillating crystals are proposed and studied as an alternative to $3 \mathrm{He}$. The authors present an overview of crystals able to detect the neutron energy spectra for the radioactive source identification. The high costs play a determinant role in this kind of scintillator, pushing the research to develop new organic or inorganic crystals. Among the organic crystals, stilbene has played a fundamental role to date. Moreover, to detect the different energy levels, multidetector systems and composite detectors are fundamental. Examples and future perspectives are also shown.

The paper by Maddalena et al. [12] reviews the scintillation mechanism, materials and main scintillation parameters. It focuses mainly on the organic and inorganic scintillator crystals' LY and response time. Among the inorganics, high LY and fast response is found in $\mathrm{Ce}^{3+}, \mathrm{Pr}^{3+}$ and $\mathrm{Nd}^{3+}$ lanthanide-doped scintillators, but the maximum LY is found in $\mathrm{Eu}^{3+}$-doped $\mathrm{SrI}_{2}$, reaching $10^{5}$ photons/MeV. Problems can arise from the difficulties and costs to produce lanthanide-doped crystals. The authors suggest, as a very promising alternative, lead halide perovskites that can reach high LY due to the very small bandgap, which is lower than $2 \mathrm{eV}$. A large section of the paper discusses the fabrication of nanostructured scintillators, in particular, perovskite-based ones. In fact, two-dimensional perovskite, nanotechnology and nanocrystal synthesis can improve efficiency and decrease the production costs. 
Theoretical studies, modelling and simulation pave the way for in-depth knowledge of scintillators and design of detectors to be attained. The GATE Monte Carlo Toolkit was used to study the effect of thickness and reflectors on the scintillation efficiency. In the paper by Ricci et al. [13], a small-animal gamma-optical camera, named " $\gamma$-eye", was studied by GATE. The simulation compares CsI:Na crystals with different thicknesses (from $4 \mathrm{~mm}$ to $6 \mathrm{~mm}$ ) wrapped with different reflectors. This study evaluates sensitivity, light output and spatial resolution of the various crystals. The comparison to experimental data confirmed the reliability of the simulation. Moreover, the results provide evidence that the thicker crystal presents higher sensitivity, but it results in a lower spatial resolution. On the other hand, the black tape wrapping improves the spatial resolution with respect to that of the standard $\mathrm{TiO}_{2}$ white reflector. This can be explained in terms of light spread over a higher angle.

A theoretical effort towards the understanding of the Decay Time mechanism is given by the papers of Davì [14], that, with originality, uses the mechanics of structured continua to model the scintillating crystals. The study extends and recalls, as special cases, well-known kinetic and diffusive phenomenological models and allows for constitutive relations which can also account for mechanical stress, electromagnetic interactions and crystal defects. The results obtained show that the Decay Time can be estimated when the excitation carriers' mobility or the structure and the parameters of the recombination mechanism are known. With this model, it is shown how the Decay Time estimates for $\mathrm{NaI}: \mathrm{Tl}, \mathrm{CaF}_{2}, \mathrm{Gd}_{2} \mathrm{SiO}_{5}: \mathrm{Ce}$ (GSO:Ce), and $\mathrm{LaCl}_{3}: \mathrm{Ce}$ are consistent with the known available data. The advantage of this model is that it is based on a limited set of known experimentally measured parameters.

The contributions of this Special Issue range from quality control to the theoretical approach, providing a large spectrum of reviews, not only on the state-of-the-art, but also proposing new ideas and insight in the direction of obtaining the most efficient scintillator crystals. Firstly, models and techniques for a fast and substantial characterization are provided, then a variety of scintillating crystals are reviewed (including organic and nanostructured crystals) with different purposes, concluding with simulations and theoretical efforts. The goal is to pave the way to low-cost and high-performance scintillating crystals.

Conflicts of Interest: The authors declare no conflict of interest.

\section{References}

1. Lecoq, P. Development of new scintillators for medical applications. Nucl. Instrum. Methods Phys. Res. Sect. A Accel. Spectrometers Detect. Assoc. Equip. 2016, 809, 130-139. [CrossRef]

2. Lecoq, P.; Gektin, A.; Korzhik, M. Inorganic Scintillators for Detector Systems; Particle Acceleration and Detection; Springer International Publishing: Cham, Switzerland, 2017.

3. Apollinari, G.; Bejar Alonso, I.; Bruning, O.; Lamont, M.; Rossi, L. High-Luminosity Large Hadron Collider (HL-LHC): Preliminary Design Report; CERN Yellow Reports: Monographs; CERN: Geneva, Switzerland, 2015.

4. Lebeau, M.; Gobbi, L.; Majni, G.; Paone, N.; Pietroni, P.; Rinaldi, D. Mapping residual stresses in $\mathrm{PbWO}_{4}$ crystals using photo-elastic analysis. Nucl. Instrum. Methods Phys. Res. Sect. A Accel. Spectrometers Detect. Assoc. Equip. 2005, 537. [CrossRef]

5. Rinaldi, D.; Montalto, L.; Lebeau, M.; Mengucci, P. Influence of a Surface Finishing Method on Light Collection Behaviour of PWO Scintillator Crystals. Photonics 2018, 5, 47. [CrossRef]

6. Gundacker, S.; Auffray, E.; Pauwels, K.; Lecoq, P. Measurement of intrinsic rise times for various L(Y)SO and LuAG scintillators with a general study of prompt photons to achieve 10 ps in TOF-PET. Phys. Med. Biol. 2016, 61, 2802-2837. [CrossRef] [PubMed]

7. Lecoq, P. Pushing the Limits in Time-of-Flight PET Imaging. IEEE Trans. Radiat. Plasma Med. Sci. 2017, 1, 473-485. [CrossRef]

8. Monachesi, E.; Dezi, A.; D’Ignazio, M.; Scalise, L.; Montalto, L.; Paone, N.; Rinaldi, D.; Loudos, G.; David, S. Comparative Evaluation of Cesium Iodide Scintillators Coupled to a Silicon Photomultiplier (SiPM): Effect of Thickness and Doping on the Scintillators. J. Phys. Conf. Ser. 2017, 931, 012013. [CrossRef] 
9. Montalto, L.; Natali, P.P.; Scalise, L.; Paone, N.; Davì, F.; Rinaldi, D.; Barucca, G.; Mengucci, P. Quality Control and Structural Assessment of Anisotropic Scintillating Crystals. Crystals 2019, 9, 376. [CrossRef]

10. Natali, P.P.; Montalto, L.; Daví, F.; Mengucci, P.; Ciriaco, A.; Paone, N.; Rinaldi, D. Theoretical and experimental evaluation of piezo-optic parameters and photoelastic constant in tetragonal PWO. Appl. Opt. 2018, 57, 730. [CrossRef] [PubMed]

11. Cieślak, M.J.; Gamage, K.A.A.; Glover, R. Critical Review of Scintillating Crystals for Neutron Detection. Crystals 2019, 9, 480. [CrossRef]

12. Maddalena, F.; Tjahjana, L.; Xie, A.; Arramel, A.; Zeng, S.; Wang, H.; Coquet, P.; Drozdowski, W.; Dujardin, C.; Dang, C.; et al. Inorganic, Organic, and Perovskite Halides with Nanotechnology for High-Light Yield Xand $\gamma$-ray Scintillators. Crystals 2019, 9, 88. [CrossRef]

13. Ricci, R.; Kostou, T.; Chatzipapas, K.; Fysikopoulos, E.; Loudos, G.; Montalto, L.; Scalise, L.; Rinaldi, D.; David, S. Monte Carlo Optical Simulations of a Small FoV Gamma Camera. Effect of Scintillator Thicknesses and Septa Materials. Crystals 2019, 9, 398. [CrossRef]

14. Daví, F. Decay Time Estimates by a Continuum Model for Inorganic Scintillators. Crystals 2019, 9, 41. [CrossRef]

(C) 2020 by the authors. Licensee MDPI, Basel, Switzerland. This article is an open access article distributed under the terms and conditions of the Creative Commons Attribution (CC BY) license (http://creativecommons.org/licenses/by/4.0/). 